\title{
Stem Cells and Their Niches: Integrated Units That Maintain Drosophila Tissues
}

\author{
A.C. Spradling, T. Nystul, D. Lighthouse, L. Morris, D. Fox, R. Cox, T. Tootle, \\ R. FREDERICK, AND A. SKORA \\ Howard Hughes Medical Institute Research Laboratories, Department of Embryology, \\ Carnegie Institution of Washington, Baltimore, Maryland 21218
}

\begin{abstract}
The genetic analysis of four distinct Drosophila stem cells and their niches has revealed principles of stem cell biology that are likely to apply widely. A stem cell and its niche act together as integral parts of a system that supplies replacement cells when and where they are needed within a tissue. Stem cell/niche units are highly regulated and continue to operate despite the periodic turnover and replacement of all of their component cells. To successfully respond to tissue needs, these units receive and process a wide range of local and systemic information. A stem cell alone would be no more use at this task than an isolated neuron. It is only when integrated into a system of multiple interacting cells (the niche) that stem cells achieve the capacity to serve as the fundamental units of tissue homeostasis and repair.
\end{abstract}

Adult metazoan tissues maintain a high level of functionality by routinely replacing their component cells. Stably differentiated cells may temporarily transition to a state where they can divide and produce daughters like themselves, without reiterating their entire process of developmental specification. More commonly, regenerative potential is based on tissue stem cells. During development, many tissues set aside a relatively small number of specific cells that maintain themselves in a less differentiated state while generating daughter cells that complete development in an adult milieu. Some of the most accessible and best-understood adult metazoan stem cells are found in the gonads and gut of Drosophila. The powerful genetics and relatively simple tissue architecture of this model organism has made it possible to identify stem cells in situ and to analyze their behavior and molecular regulation, providing useful insights for stem cell biology in general (for review, see Morrison and Spradling 2008).

One of the most important contributions of Drosophila stem cell studies has been to document the central role of the stem cell niche. Stem cell behavior in vivo depends on the local microenvironment - the stem cell niche (Xie and Spradling 2000). Although some stem cells can be isolated (Bryder et al. 2006) and cultured in vitro (KanatsuShinohara et al. 2003; Niki and Mahowald 2003), they must still be returned to a tissue niche for normal activity. The defining characteristic of niches is that they are required for stem cell maintenance, i.e., for self-renewing divisions that also generate new tissue cells. In the absence of this natural milieu, or the artificial mixes of growth factors found in stem cell culture media, stem cells begin to differentiate. Making stem cells dependent on factors that are not readily available throughout the tissue, but only found in isolated "niches," may be a strategy to tightly align stem cell activity with tissue needs and limit unregulated stem cell proliferation, which poses an obvious danger. Once a tissue's niches are filled, any additional potential stem cells that arise by somatic muta- tion or failed differentiation would be unable to sustain themselves. Stem cell preservation provides the defining test for a candidate stem cell niche: It must maintain introduced competent cells as stem cells, cells that would differentiate or die at all other locations.

Here, we review recent progress and argue that stem cell/niche systems do more than store stem cells and limit overproliferation. They must determine the number of replacement cells and their states of differentiation needed at each location. Answering these questions requires the stem cell/niche unit to receive and process inputs from hormones, intercellular signals, mechanical stresses, sensory information, etc. Thus, the task of the stem cell and its niche is not unlike that of a neural ganglion. A stem cell alone would be of no more use than an isolated neuron. It is only when integrated into an information processing system comprising multiple linked cells and cell types (the niche) do stem cells achieve the capacity to serve as a fundamental unit of tissue homeostasis and repair.

\section{METHODS}

Drosophila strains. Clonal analysis was performed essentially as described by Nystul and Spradling (2007). $\beta$ galactosidase $\left(\beta\right.$-gal $\left.{ }^{+}\right)$-positive clones (Harrison and Perrimon 1993) were generated using $y w, p\left[(h s F l p)^{12}, r y^{+}\right]$; $X .15 .29$, and $y w ; X .15 .33$. Dual-marked clones were induced using $y^{122}$, hsFlp; FRT 42D tub-lacZ/cyo; TM2/ $T M 6 B$ and $w$; and FRT 42D, Ubi-GFP/CyO (Bloomington). For further information, see FlyBase (http://flybase.bio. indiana.edu).

Immunofluoresence microscopy. Immunofluoresence microscopy was performed essentially as described in Nystul and Spradling (2007). Ovaries dissected in Grace's medium were fixed in 4\% paraformaldehyde for 10 minutes, rinsed with $0.2 \%$ Triton X-100 in phosphate-buffered 
saline (1x PBST) and 1x PBST $+5 \%$ normal goat serum, and then incubated with primary antibody (overnight at $4^{\circ} \mathrm{C}$ ). After washing in $1 \times$ PBST ( $3 \times 20$ minutes), tissue was incubated with secondary antibody (1x PBST, $0.5 \%$ bovine serum albumin [BSA], and 60 minutes), and washed in $1 \times$ PBST ( $3 \times 20$ minutes) and $1 \times$ PBS $(3 \times 20$ minutes). Slides were stained with DAPI (4'-6-diamidino2-phenylindole) and mounted with Vectashield (Vector).

Electron microscopy. Electron microscopy was performed essentially as described by Cox and Spradling (2003). Tissue isolated in Grace's media was fixed for 1 hour $\left(1 \%\right.$ gluteraldehyde, $1 \% \mathrm{OsO}_{4}, 0.1 \mathrm{M}$ cacodylate buffer, $2 \mathrm{~mm} \mathrm{Ca}$ at $\mathrm{pH} 7.5$ ). Following washes in cacodylate buffer ( $3 \times 5$ minutes) the tissue was embedded in agarose at $55^{\circ} \mathrm{C}$, rinsed in $0.05 \mathrm{M}$ maleate $(\mathrm{pH} 6.5)(1 \times 5$ minutes), and incubated in $0.5 \%$ uranyl acetate, $0.05 \mathrm{M}$ maleate ( $1 \times 1.5$ hours). Tissue was then dehydrated through ethanol (35\% 2× 5 minutes, 50\% 10 minutes, $75 \% 10$ minutes, $95 \% 10$ minutes, $100 \% 3 \times 10$ minutes), incubated in propylene oxide ( $2 \times 10$ minutes), and 1:1 propylene oxide:resin (Epon 812:Quetol 651(2:1):1\% silicone 200, $2 \%$ benzyldimethylamine [BDMA]) ( $1 \times 1$ hour). Resin was changed ( $3 \times 1$ hour) and then allowed to polymerize at $50^{\circ} \mathrm{C}\left(>8\right.$ hours) and then at $70^{\circ} \mathrm{C}(>8$ hours). Images were captured with a Phillips Tecnai 12 microscope and recorded with a GATAN multiscan CCD (charged-coupled device) camera using Digital Micrograph software.
Lineage analysis. Lineage analysis, as described by Nystul and Spradling (2007), was performed by generating either $\beta$-gal ${ }^{+}$clones or dual-marked clones (see Drosophila strains). $\mathrm{F}_{1}$ flies that contained hsFlp and the two appropriate FRT-containing chromosomes were maintained with fresh food at $25^{\circ} \mathrm{C}$, and mitotic clones were generated by heat shocks $\left(37^{\circ} \mathrm{C} \times 60\right.$ minutes $)$. To assay for nondividing FSC niche cells, this treatment was repeated once a day for 3-5 days.

\section{RESULTS}

\section{Anatomically Fixed Niches: The Germ-line Stem Cell Niche}

Early studies of Drosophila niches focused on the female and male germ-line stem cells (GSCs) (Xie and Spradling 2000; for review, see Fuller and Spradling 2007), because these stem cells and niches are among the simplest and most accessible in all of biology. The location of the female GSC niche at the blind end of each tubular ovariole fulfilled many preconceptions of what a niche should be like (Fig. 1). Here, the terminal filament and cap cells define a unique tissue microenvironment that activates the Dpp (Decapentaplegic) signaling pathway within resident germ cells to much higher levels than cells just one cell diameter away (Chen and McKearin 2003; Song et al. 2004). No other region of the normal

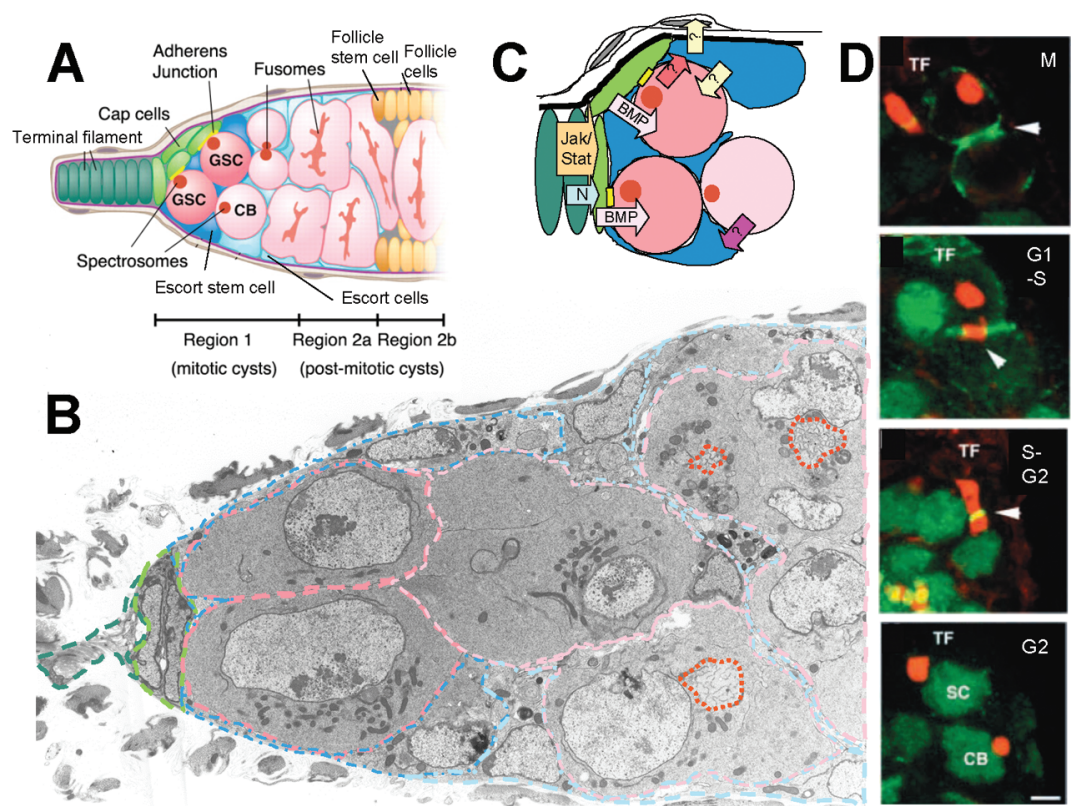

Figure 1. The germ-line stem cell niche. (A) Diagram of a Drosophila germarium (Nystul and Spradling 2007) showing the germ-line stem cells (GSC), escort stem cells, and stromal cells that make up the GSC niche located just posterior to the cap cells (light green). Follicle stem cells (gold) are found at the junction of regions $2 \mathrm{a}$ and $2 \mathrm{~b}$, where cysts (pink) begin to move in single file. $(B)$ Electron micrograph of the GSC niche region. The cells have been outlined in colors that correspond to the diagram above. Cross sections of the fusome are outlined in red. (C) Known and proposed signals that mediate niche/stem cell regulation. Cells are colored as in $A$. (Thick black line) Basement membrane; (gray) ovarian (muscle) sheath. (D) Fusome behavior during the GSC cell cycle. At M phase, the spectrosome is entirely within the anterior cell (closer to the terminal filament, TF). The cytokinesis furrow is beginning to form (arrowhead). During $\mathrm{G}_{1}-\mathrm{S}$, new fusome material grows within the arrested cytokinesis furrow (arrowhead). During late $\mathrm{S}-\mathrm{G}_{2}$, the two segments of fusome material fuse; however, more always is found within the GSC than in the cystoblast. During $\mathrm{G}_{2}$, the cytokinesis furrow separating the GSC from the cystoblast (CB) finally closes, leaving the two cells with differing amounts of fusome. (Red) Hts protein; (green) Anilin. (Modified from deCuevas and Spradling 1998.) 
germarium induces such strong pathway activation (Kai and Spradling 2003), and only this high level is sufficient to repress the bag-of-marbles (bam) gene and suppress GSC differentiation. Remarkably, a germ cell daughter derived from the division of a nearby GSC or a cystocyte up to the eight-cell stage, cells that would differentiate outside of the niche, can dedifferentiate and become functional GSCs if they gain niche access (Brawley and Matunis 2004; Kai and Spradling 2004).

If the niche was simply a small zone capable of holding a GSC in place and blocking its differentiation by regulating $\mathrm{bam}$, it could not adequately function. A major role of the niche is to regulate cell production, not simply generate daughters continuously. Stem cell division responds directly to nutritional status (DrummondBarbosa and Spradling 2001) and the actual rate of egg deposition. Nutritional levels are sensed by GSCs and their progeny using the insulin-signaling pathway (Drummond-Barbosa and Spradling 2001; LeFever and Drummond-Barbosa 2005). How oviposition is sensed and whether oviposition from specific ovarioles can be distinguished remain unknown, but they may involve prostaglandin signaling (Tootle and Spradling 2008). The niche probably also mediates interactions among individual GSCs and facilitates the replacement of some GSCs by the daughters of others (Xie and Spradling 2000; Yamashita et al. 2007; Jin et al. 2008). The functioning of both male and female niches and their stem cells declines with age, because of changes in niche cells, niche signals, and stem cell responsiveness (Wallenfang et al. 2006; Boyle et al. 2007; Pan et al. 2007).

Given this sophistication, it is not surprising that more cells and signals participate in niche operation than originally appreciated. A second type of stem cell in females, the escort stem cell (ESC), also contacts cap cells and covers most of the GSC surface (Decotto and Spradling 2005). ESCs maintain the population of escort cells (also known as inner germarium sheath cells) that surround developing germ cells before they acquire a follicle cell layer. JAK/STAT signaling has an important role in the female niche, as well as the male niche (Decotto and Spradling 2005; López-Oneiva et al. 2008; Wang et al. 2008). JAK/STAT ligand levels are high in the terminal filament, and these cells signal to cap cells and ESCs, which relay the critical bone morphogenetic protein (BMP) signals, i.e., dpp for GSC division and maintenance and possibly other signals to GSCs and muscle sheath cells as well (Fig. 1C).

Even after the niche has determined that it is time to stimulate GSC division and the distal daughter has separated and up-regulated bam transcription, it remains unclear how this leads to cystoblast differentiation. GSCs express transcripts required for growth, but they repress nearly all genes associated with embryonic and tissue differentiation (Kai et al. 2005), much like ES cells. Premature expression of differentiation genes is repressed at multiple levels, including extensive translational control mediated by products of the pumilio, nanos, CPEB, and microRNA (miRNA) genes (Spradling et al. 1997; Jin and Xie 2007; Park et al. 2007; Neumüller et al. 2008). One theory is that Bam binds to differentiation gene
mRNAs to counteract the translational repression mediated by Nanos and Pumilio (Chen and McKearin 2005; Szakmary et al. 2005). However, it remains possible that translational up-regulation is an effect of cyst differentiation, rather than a direct cause, and that Bam acts by a different mechanism.

\section{The Role of the Fusome}

A better understanding of the fusome (Fig. 1B), an organelle rich in endoplasmic reticulum (ER)-like tubules that is found in early germ cells (Spradling et al. 1997; Snapp et al. 2004), might help to resolve how bam controls GSC differentiation. A specific subset of Bam protein appears to reside within the fusome, and a bam null allele greatly reduces the number of fusome vesicles (McKearin and Ohlstein 1995). Moreover, fusome protein Ter94, related to the yeast ER vesicle fusion mediator $\mathrm{Cdc} 48$, interacts with Bam and depends on Bam for its normal fusome enrichment (León and McKearin 1999). It is plausible that after separation from the GSC is complete, Bam catalyzes a process of ER maturation within the fusome that initiates cystoblast development. So far, however, the nature of any molecular differences between the round fusome within the GSC (which is called the spectrosome), and the cystoblast fusome remains unclear (Spradling et al. 1997). To learn more about the role of the fusome in GSC maintenance and cystoblast specification, we developed a large collection of protein trap strains (Buszczak et al. 2007) and used them to identify new fusome components (Lighthouse et al. 2008).

The fusome synchronizes the cystocyte cell cycles and induces cleavage-like divisions (Spradling et al. 1997; Lilly et al. 2000), but a function in GSCs has not been determined previously. We analyzed GSC clones of mutations in many of the newly identified fusome components to determine whether fusome structure, GSC behavior, or cystoblast development was defective. Loss of several genes, including tmod, encoding the cytoskeletal protein tropomodulin; $\mathrm{Fer} 1 \mathrm{HCH}$, encoding the ironbinding protein ferritin (heavy chain); and scrib, encoding an epithelial polarity protein, has no effect on fusome morphology (not shown) or on GSC lifetime (Fig. 2A). In contrast, clones disrupting production of the small GTPase Rab11, a key component of the recycling endosome, drastically alter fusome morphology, disrupt normal cyst formation, and greatly accelerate GSC loss (Fig. 2B). Fusomes within the rab11 mutant GSCs contain greatly reduced numbers of ER-like tubules (Fig. 2C). Because we could find no changes in the adherens junctions between GSCs and cap cells, we suggest that the alterations in the fusome disrupt a germ cell signal whose production depends on fusome vesicle maturation, leading to changes that disrupt cyst development and destabilize the GSCs (Lighthouse et al. 2008). The nature of this fusome-dependent signal is currently under investigation.

\section{Dynamic Niches: The Follicle Stem Cell Niche}

Studies of GSC niches have provided significant insight into the development, function, and aging of stem cells 

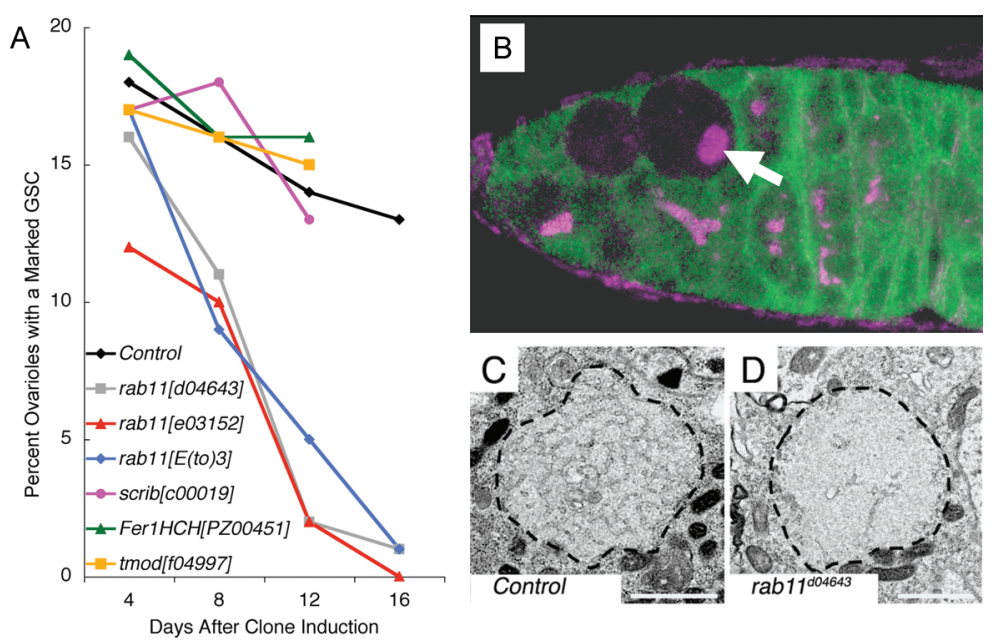

Figure 2. Rab11 is required to maintain GSCs and for normal cyst formation. (A) Rab11 mutations accelerate GSC loss. The graph shows the rate of loss of marked GSCs bearing the indicated mutations or no mutation (control). All three tested rab11 mutations greatly accelerate the rate of GSC loss, compared to wild type or the other gene mutations tested. $(B)$ Germarium containing germ-line clones rab11[d04643] indicated by the absence of LacZ staining (green). One mutant GSC is present, along with a cyst that has developed aberrantly, and contains a large mass of fusome material in one oversized cell (arrow). (C) Electron micrograph of a normal round fusome from wild-type GSC, showing the presence of ER-like vesicles. $(D)$ Electron micrograph of round fusome from a rab11[d04643] GSC. The number of ER-like vesicles is greatly reduced. (Modified from Lighthouse et al. 2008.)

interacting with their niches in an intact tissue. Niches based on similar specialized stroma may also occur in mammals, for example, within intestinal crypts, the bulge region of hair follicles, or along the surface of muscle fibers and seminiferous tubules. Some tissues, such as mammalian interfollicular epidermis, lack obvious candidate regions, however. It remains controversial whether specific stem cells and niches even exist in this tissue (Clayton et al. 2007). The Drosophila ovary provides a potential model for understanding mammalian epithelial stem cells and their niches. Each developing Drosophila follicle arises from an epithelial layer in the germarium produced by two epithelial stem cells (FSCs) that so closely resemble their daughters that they were never distinguished by classical histology (Spradling et al. 1997).

Each germarium contains exactly two FSCs and they each reside in a separate small niche (Nystul and Spradling 2007). However, FSC niches differ sharply from those previously characterized for GSCs. First, they lack permanent, nondividing stromal partner cells, the cells around which GSC niches are built. We demonstrated this using a Drosophila strain in which all cells in the germarium are marked with both green fluorescent protein (GFP) and LacZ (Fig. 3A'). Upon heat shock, stem cells undergo recombination and produce daughters that are either $\mathrm{LacZ}^{+}$or $\mathrm{GFP}^{+}$but not both. Following multiple heat shocks, we found that all of the cells surrounding the FSCs could become unilabeled, whereas the cap cells of the GSC niche always retained both markers (Fig. 3A). Therefore, all of the FSC's neighbors are transient, moving cells that will either die or depart to join new follicles. There are no cap cell equivalents. Yet despite this lack of permanent cells, FSCs remain in the same relative location, at the position where cysts form a single file, lose their escort cells, and begin to acquire fol- licle cell replacements (known as the region $2 \mathrm{a} / 2 \mathrm{~b}$ border). Further analyses show that FSCs in this region contact the basement membrane, the escort cells covering approaching cysts, and several FSC daughter cells, but they never touch germ cells (Fig. 3B).

Not only are the cellular components of the FSC niche transitory, but its location is not anatomically fixed. The exact number of cysts in each region can vary with time, but there is always a characteristic region $2 \mathrm{a} / 2 \mathrm{~b}$ border. Cysts upstream of this boundary are covered with escort cells, whereas region $2 \mathrm{~b}$ cysts span the width of the germarium and are covered with follicle cells. The two FSCs are always found in the same relative position at this boundary (Fig. 3C). We propose that this type of "dynamic niche" in which stem cells are maintained in a defined but constantly changing microenvironment represents a common and widespread type of tissue niche.

The dynamic niche is our term for this cyclically changing microenvironment amid the moving, developing cells that surround the FSC. Rather than residing in a fixed microenvironment and responding to a relatively small number of signals, FSCs likely experience a 24-hour cycle of microenvironments. By making appropriate choices at each juncture, the sequence eventually repeats. This looping sequence of microevironments would be generated by the changing contacts between the approaching and leaving cyst and its escort cells, apoptosis of escort cells, and inward migration of young follicle cells adjacent to the FSCs. Generating and maintaining such a dynamic signaling milieu seems complex and subject to perturbations that might cause the resident stem cells to be lost or to be programmed incorrectly. However, such a system may also offer advantages over static niches in assuring that the resident stem cell remains perfectly in tune with the needs of the tissue it serves. 

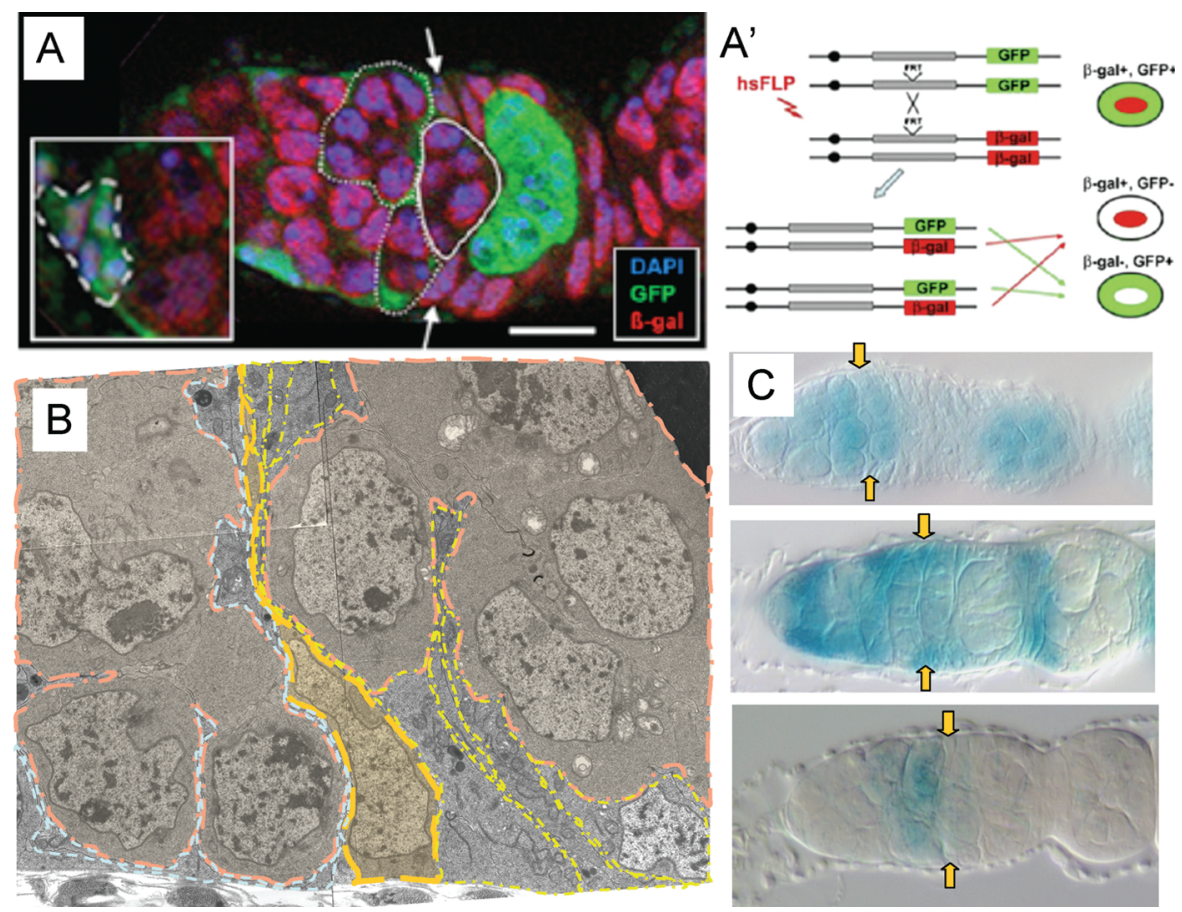

Figure 3. The dynamic FSC niche lacks fixed stromal cells. (A) A strain bearing a dual clone-marking system (A') was subjected to multiple heat shocks to label all cells that are cycling within the anterior germarium. The arrows point to the two FSCs, and the absence of any cells bearing both the LacZ and GFP markers (such as the nondividing cap cells, inset) shows that no nondividing cells border the FSCs. (B) An electron micrograph of an FSC (gold outline) in its normal microenvironment shows that it contacts only escort cells on upstream cysts, its own follicle cell progeny (yellow outlines), and those of its partner stem cell on the other side of the germarium (not visible). The FSC does contact a region of basement membrane, but it apparently does not directly touch any germ cells. $(C)$ Three micrographs of wild-type Drosophila germaria are shown to illustrate the variation in the position of the FSCs (arrows) relative to the germaria as a whole. Some variation is due to differences in the number of $2 a$ and $2 b$ cysts between germaria; however, additional variation is intrinsic due to the movements that take place over a 24 -hour cycle as cysts move into single file at the region $2 \mathrm{a} / 2 \mathrm{~b}$ border. X-gal staining (blue) is not comparable between panels. (A, Modified from Nystul and Spradling 2007.)

\section{Stem Cell Dynamics: Both Stem Cells and Niche Cells Are Frequently Replaced}

The dynamic FSC niche maintains stem cell activity, despite undergoing continuous morphological change: the loss of old niche cells downstream and the addition of new ones upstream. This is reminiscent of the stem cell replacement observed previously within the stable GSC niche. Although individual cap cells and terminal filament cells do not change with time, individual GSCs frequently turn over and are replaced (Margolis and Spradling 1995; Xie and Spradling 2000). There appears to be no loss of stem cell/niche functionality associated with this stem cell replacement, and indeed, ongoing replacement has been postulated to depend on competition and to ensure that highly functional cells serve as stem cells (Nystul and Spradling 2007; Jin et al. 2008). Thus, the maintenance of functionality despite frequent substitution of constituent cells is a prominent aspect of all studied stem cell/niche systems.

In the dynamic niche, both niche cells and stem cells are subject to replacement. In fact, in the FSC niche, external niche cells are undergoing cyclic replacement with the passage of each cyst, whereas individual FSCs turn over about once every 30 divisions (Nystul and Spradling 2007). Thus, stem cell/niche systems are self- correcting systems that maintain their critical functions even though all of their constituent cells are ultimately dispensable. They are like the wave, not the moving particles of the underlying medium.

\section{Talking Back: Intestinal Stem Cells and Daughter Cell Programming}

We have emphasized the importance of the niche in controlling stem cell behavior and adapting their activity to the needs of the tissue and organism. However, study of another Drosophila stem cell provides clear indications that stem cells as well as niche cells participate in regulatory interactions. The Drosophila posterior midgut is maintained throughout adulthood by $800-1000$ intestinal stem cells (ISCs) (Michelli and Perrimon 2006; Ohlstein and Spradling 2006) that resemble mouse intestinal stem cells (Barker et al. 2007) in many important respects. Like mammalian ISCs, Drosophila stem cells are multipotent, giving rise to both enterocytes and enteroendocrine cells (Fig. 4A). In addition, ISCs in both flies and mice require Notch signaling to produce enterocytes; when Notch is mutant, an excess number of secretory (enteroendocrine) cells result. Finally, ISCs even show morphological similarities (Fig. 4B); in both species, they reside against the 

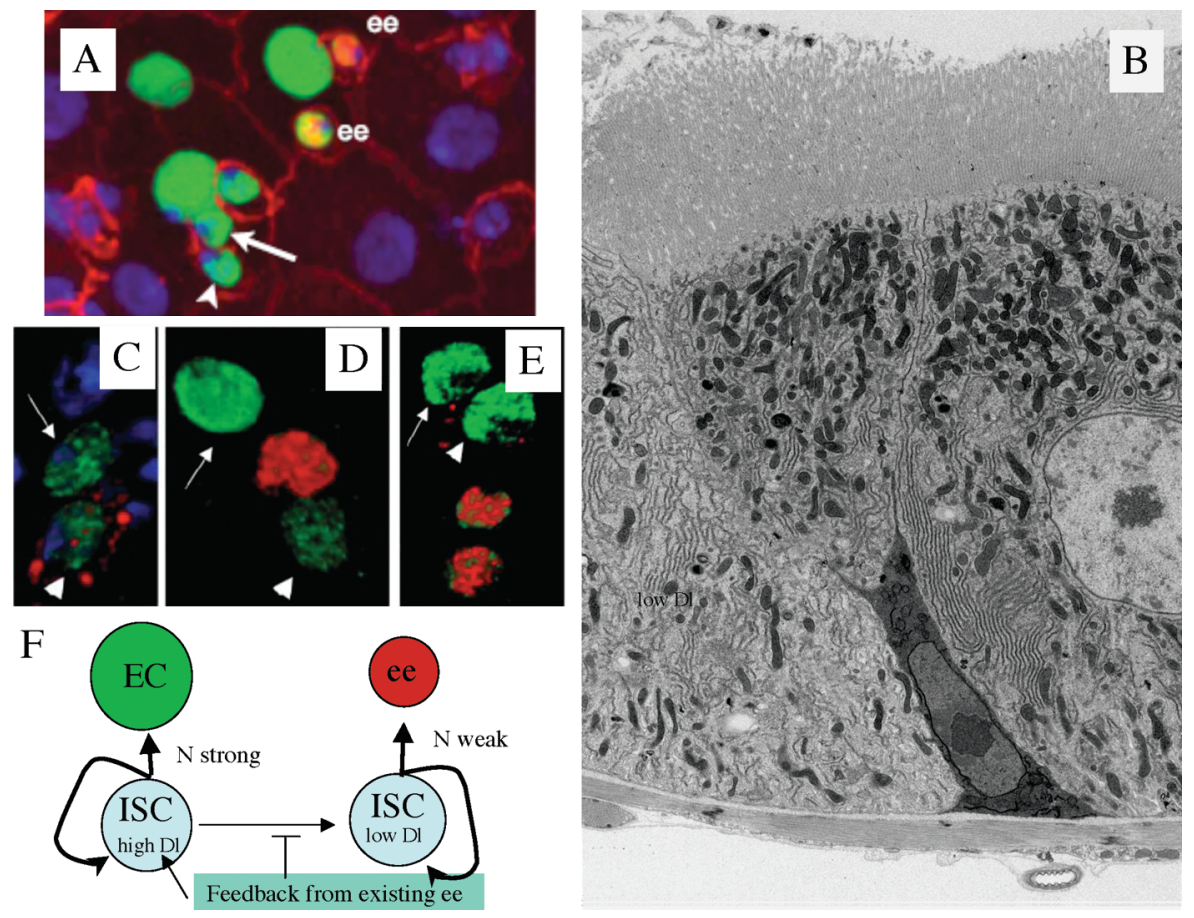

Figure 4. ISCs program their daughters by differential Notch signaling. (A) A clonally labeled ISC (arrowhead) has given rise to seven progeny (green): five enterocytes and two enteroendocrine cells (red), proving that ISCs are multipotent (from Ohlstein and Spradling 2006). (B) An electron micrograph of the adult midgut showing that like the FSC, ISCs (dark cell) contact only the basement membrane (bottom) and downstream cells such as the enterocytes shown. A cross section of a trachiole lies below the basement membrane under the ISC. (C-E) ISCs program progeny cell type via Notch signaling. (C) A two-cell clone (green) showing that ISCs (arrowhead) giving rise to enterocytes (arrow) are always rich in Delta-containing vesicles (red, cytoplasmic). (D) A three-cell clone (green) showing that when an ISC (arrowhead) switches from enterocyte (arrow) to enteroendocrine cell (red, nuclear) production, the level of Delta (red, cytoplasmic) falls drastically. $(E)$ A four-cell clone (green) showing that when an ISC (arrowhead) switches from enteroendocrine cell production to enterocyte production, Delta-rich vesicles return to the cytoplasm. $(F)$ Model of ISC programming: A feedback signal from existing enteroendocrine cells maintains high Delta levels in ISCs that causes a strong signal to be sent specifying enterocyte fate. In the absence of nearby enteroendocrine cells, Delta levels fall, and the resulting low-level Notch signal specifies enteroendocrine cell fate. ( $A-F$, Modified from Ohlstein and Spradling 2007.)

basement membrane but extend thin cytoplasmic extensions up to the vilar surface where they may receive information from the gut lumen (Ohlstein and Spradling 2006; Barker et al. 2007).

The ISC niche is less well characterized than that of the FSC, but there appear to be some interesting similarities. ISCs are not found in fixed positions within the midgut, but it remains to be determined if they are in a predictable position relative to the surrounding cells, i.e., if there is a gut equivalent to the $2 \mathrm{a} / 2 \mathrm{~b}$ boundary. They contact the basement membrane and differentiating or differentiated ISC daughters but not any permanent niche cells. One of the most interesting aspects of ISCs is their multipotency, which depends on Notch signals sent by ISCs shortly after division that program daughter cell fate. A strong Notch signal specifies the daughter to become an enterocyte (Fig. 4C,E), whereas a weak signal results in enteroendocrine differentiation (Fig. 4D) (Ohlstein and Spradling 2007). The observation that enteroendocrine cells are made in pairs and that each ISC makes no more than one pair if existing enteroendocrine cells remain nearby lead to a simple feedback model for cell-fate specification by a multipotent stem cell (Fig. 4F). Thus, in this tissue, sig- nals reporting a tissue's status may be sensed and interpreted directly by the stem cell, which then responds by specifying an appropriate type of daughter cell.

\section{DISCUSSION}

\section{Niches and Stem Cells Work as Dynamic Integrated Units}

Our studies demonstrate that both stem cells and niche cells are essential components of a regulatory system that produces new cells of the appropriate types at the times and locations needed to maintain tissue health. None of the individual cells, whether stem cells or niche cells, on their own appears to be essential for stem cell/niche function. The capacity for stem cell/niche function is not maintained due to the long life or unusual properties of any individual cell. Rather, tissue maintenance is based on the regular production of interchangeable parts and a stable program of cellular interactions, governed by reciprocal signals highly stabilized to perturbations. These stem cell/niche systems are capable of reacting to diverse situations, calculating and putting into effect appropriate responses 
including changes in division rate, movement, and cell specification. We probably need new terminology to describe this type of biological system, which likely constitutes a fundamental aspect of multicellularity.

\section{The Dynamic Niche and the Importance of Studying Stem Cells In Vivo}

There are several practical corollaries to these findings. It has too often been assumed that stem cells are sufficiently autonomous that properties such as developmental potency and division potential will be intrinsically maintained even during culture in the absence of normal neighbors and in the presence of very different signals and factors than they experience in vivo. However, this belief has yet to be documented in the case of a stem cell whose behavior in vivo has been independently determined. Stem cells have also been thought to behave in such a uniform fashion that they could be distinguished from other cells in diverse tissues using simple general properties such as a slow cell cycle. However, we see that true stem cells observed in vivo interact extensively with their neighboring cells and microenvironment in ways that differ among particular stem cells, niches, and physiological situations.

The more we know about stem cell/niche systems, the more we realize how difficult they will be to reconstruct in culture. Not only might it be difficult to find a feeder cell or soluble factors that can mimic the surface interactions between stem cell and niche, but our findings suggest that the microenvironment may need to cycle in a complex three-dimensional sequence. After in vivo study to understand the cycle, simulation might be possible, but the chances seem low that a solution can be found empirically by guesswork. For these reasons, it is now clear that stem cells should initially be identified and studied in vivo to establish their behavioral parameters. Fortunately, an ever-widening array of noninvasive cell-marking techniques are becoming available (for review, see Fox et al. 2009), as are methods for the direct observation of stem cell/niche systems in living tissue.

\section{Implications of Stem Cell/Niche Units as Dynamic Entities Mediating Homeostatic Responses}

Sustained tissue function over an extended period requires many decisions. For example, Drosophila can lay eggs at a high rate under ideal conditions, but egg production slows in many situations: circadian day, limited diet (especially protein), lack of attractive oviposition sites, etc. Thus, sensory and nutritional inputs are essential. Insulin levels are read directly by the stem cell. Neuronal input regarding oviposition sites is likely relayed to the oviducts, either by direct neuronal connection to oviduct musculature or via neurosecretion. It is not known how oviposition controls follicle development all the way back to the stem cells, but hormonal signals such as prostaglandins that have been implicated in oviposition in many species are possible candidates.

Previously, intercellular signaling and asymmetric division mechanisms have been recognized as being par- ticularly important for understanding stem cell behavior. However, if niches must cycle through a series of particular states, each inducing a response in the stem cell, then many additional cell biological mechanisms will likely affect stem cell/niche function. Cell migration, apoptosis, shape change, extracellular matrix characteristics, and many other mechanisms might all affect stem cell/niche choreography.

\section{The Dynamic Niche and the Role of Stroma in Cancer}

The idea that epithelial stem cells read and react to complex sequences of neighboring cell activity has several potentially significant implications. Abnormalities in stromal cells contribute to uncoordinated growth and may act as a stepping-stone to cancer. Repeated wounding increases the chance of cancer development, but this is often ascribed to the elevated number of divisions that stem cells and early progenitors undergo to repair these insults. However, repeated wounding and scar formation may also disrupt the ability of cells to move normally and might change the way moving cells contact other cells in the affected region. Our findings suggest that this alone might affect stem cell programming, leading to inappropriate cycles of activity and metaplasia. To test these ideas, it will be essential to understand in detail the complete cycle of a normal dynamic niche and to study the effects of perturbations at each point in the program. In addition, we will need to learn whether these interactions are unique to the niche and stem cell or whether nonstem cell populations are guided by similar cues and might be capable of correcting or interfering with normal stem cell/niche programming.

\section{Implications of Stem Cell/Niche Interactions for Stem Cell Therapy}

Our growing realization that stem cells act in conjunction with tissue niches as integrated units for tissue maintenance has implications for stem cell therapy using cells grown in vitro. Unless the target tissue has a large supply of empty and compatible niches, it is unlikely that the introduced cells will persist or function as stem cells or that any therapeutic effect will be more than short-lived. Niches sometimes appear to be available when external factors such as radiation, infection, or autoimmunity preferentially destroy stem cells. However, as normal Drosophila adults age, their niches remain filled with stem cells, despite regular replacement of individual cells. In very old animals, niches may no longer produce high enough levels of signal to remain functional (Boyle et al. 2007; Pan et al. 2007). However, the condition most amenable to stem cell therapy, i.e., the presence of functional niches unoccupied by stem cells, has not been observed during normal Drosophila development.

What about the possibility of supplying cells farther downstream in the lineage? Here again, our studies suggest that knowledge of normal tissue biology will likely be needed to guide cell transplantation. In the stem cell lineages we studied, cell specification often begins close 
to or within the niche; ectopic cells may not differentiate or function properly without such a start. In a tissue such as the intestine with its common, dispersed stem cells, replacement cells are probably generated almost on site from the stem cells closest to the lesion and differentiate as part of an interacting group of cells. Cell therapy would be most promising in a tissue where replacement cells travel some distance from their site of origin and have evolved an ability to find, identify, and repair damage. Added cells will likely have to closely match some normal process that occurs in vivo, and it will be crucially important to identify those situations and understand the conditions required for repair to take place.

Consequently, much more emphasis in stem cell research is needed on the tissue niches and on the behavior of downstream cells in normal tissue repair. Medical conditions need to be evaluated in terms of the type of defects in cell replacement that have caused them and the subsequent consequences: loss of stem cells, loss of niches, disordering of tissue architecture, etc. Many conditions may be treatable by addressing changes in the niche, rather than by adding stem cells. Tissue homeostasis is an equal partnership between stem cells and their niches. Consequently, stem cell therapy needs to become a more balanced field in which the state of the host tissue is given the same consideration as the nature of the cells to be added.

\section{ACKNOWLEDGMENTS}

We thank many former members of the Spradling lab including Drs. Ben Ohlstein and Michael Buszczak, whose work contributed greatly to the development of these ideas. The authors are also grateful to Dianne Williams, Shelley Paterno, Megan Kutzer, and Vanessa Damm for technical assistance. Mike Sepanski provided expert help with electron microscopy, and Mamuhd Siddiqi assisted with 3D image reconstruction and timelapse micrography. This work was supported by the Howard Hughes Medical Institute and the Carnegie Institution of Washington.

\section{REFERENCES}

Barker, N., van Es, J.H., Kuipers, J., Kujala, P., van den Born, M., Cozijnsen, M., Haegebarth, A., Korving, J., Begthel, H., Peters, P.J., and Clevers, H. 2007. Identification of stem cells in small intestine and colon by marker gene Lgr5. Nature 449: 1003-1007.

Boyle, M., Wong, C., Rocha, M., and Jones, D.L. 2007. Decline in self-renewal factors contributes to aging of the stem cell niche. Cell Stem Cell 1: 458-469.

Brawley, C. and Matunis, E. 2004. Regeneration of male germline stem cells by spermatogonial dedifferentiation in vivo. Science 304: 1331-1334.

Bryder, D., Rossi, D.J., and Weissman, I.L. 2006. Hematopoietic stem cells: The paradigmatic tissue-specific stem cell. Am. J. Pathol. 169: 338-346.

Buszczak, M., Paterno, S., Lighthouse, D., Bachman, J., Plank, J., Owen, S., Skora, A., Nystul, T., Ohlstein, B., Allen, A., et al. 2007. The Carnegie protein trap library: A versatile tool for Drosophila developmental studies. Genetics 175: 1505-1531.

Chen, D. and McKearin, D. 2003. Dpp signaling silences bam transcription directly to establish asymmetric divisions of germline stem cells. Curr. Biol. 13: 1786-1791.
Chen, D. and McKearin, D. 2005. Gene circuitry controlling a stem cell niche. Curr. Biol. 15: 179-184.

Clayton, E., Doupe, D.P., Klein, A.M., Winton, D.J., Simons, B.D., and Jones, P.H. 2007. A single type of progenitor cell maintains normal epidermis. Nature 446: 185-189.

Cox, R. and Spradling, A.C. 2003. A Balbiani body and the fusome mediate mitochondrial inheritance during Drosophila oogenesis. Development 130: 1579-1590.

Decotto, E. and Spradling, A.C. 2005. The male and female Drosophila germline stem cell niche: Similar cells and signals. Dev. Cell 9: 501-510.

deCuevas, M. and Spradling, A.C. 1998. Morphogenesis of the fusome and its implications for oocyte specification. Development 125: 2781-2789.

Drummond-Barbosa, D. and Spradling, A.C. 2001. Stem cells and their progeny respond to nutritional changes during Drosophila oogenesis. Dev. Biol. 231: 265-278.

Fox, D., Morris, L., Nystul, D., and Spradling, A.C. 2009. Analysis of stem cells by lineage analysis. StemBook (in press).

Fuller, M.T. and Spradling, A.C. 2007. Male and female Drosophila germline stem cells: Two versions of immortality. Science 316: 402-404.

Harrison, D. and Perrimon, N. 1993. A simple and efficient generation of marked clones in Drosophila. Curr. Biol. 3: 424-433.

Jin, Z. and Xie, T. 2007. Dcr-1 maintains Drosophila ovarian stem cells. Curr. Biol. 17: 539-544.

Jin, Z., Kirilly, D., Weng, C., Kawase, E., Song, X., Smith, S., Schwartz, J., and Xie, T. 2008. Differentiation-defective stem cells outcompete normal stem cells for niche occupancy in the Drosophila ovary. Cell Stem Cell 10: 39-49.

Kai, T. and Spradling, A. 2003. An empty Drosophila stem cell niche reactivates the proliferation of ectopic cells. Proc. Natl. Acad. Sci. 100: 4633-4638.

Kai, T. and Spradling, A.C. 2004. Differentiating germ cells can revert into functional stem cells in Drosophila melanaogaster ovaries. Nature 428: 564-569.

Kai, T., Williams, D., and Spradling, A.C. 2005. The expression profile of purified Drosophila germline stem cells. Dev. Biol. 283: 486-502.

Kanatsu-Shinohara, M., Ogonuki, N., Inoue, K., Miki, H., Ogura, A., Toyokuni, S., and Shinohara, T. 2003. Long-term proliferation in culture and germline transmission of mouse male germline stem cells. Biol. Reprod. 69: 612-616.

LaFever, L. and Drummond-Barbosa, D. 2005. Direct control of germline stem cell division and cyst growth by neural insulin in Drosophila. Science 309: 1071-1073.

León, A. and McKearin, D. 1999. Identification of TER94, an AAA ATPase protein as a Bam-dependent component of the Drosophila fusome. Mol. Biol. Cell 10: 3825-3834.

Lighthouse, D., Buszczak, M., and Spradling, A.C. 2008. New components of the Drosophila fusome suggest it plays novel roles in signaling and transport. Dev. Biol. 217: 59-71.

Lilly, M., deCuevas, M., and Spradling, A.C. 2000. Cyclin A associates with the fusome during germline cyst formation in the Drosophila ovary. Dev. Biol. 218: 53-63.

López-Oneiva, L., Fernandez-Minan, A., and Gonzalez-Reyes, A. 2008. Jak/Stat signalling in niche support cells regulates dpp transcription to control germline stem cell maintenance in the Drosophila ovary. Development 135: 533-540.

Margolis, J. and Spradling, A. 1995. Identification and behaviour of epithelial stem cells in the Drosophila ovary. Development 121: 3797-3807.

McKearin, D.M. and Ohlstein, B. 1995. A role for the Drosophila Bag-of-marbles protein in the differentiation of cystoblasts from germline stem cells. Development 121: 2937-2947.

Michelli, C.A. and Perrimon, N. 2006. Evidence that stem cells reside in the adult Drosophila midgut epithelium. Nature 439: 475-479.

Morrison, S. and Spradling, A.C. 2008. Stem cells and niches: Mechanisms that promote stem cell maintenance throughout life. Cell 132: 598-611. 
Neumüller, R.A., Betschinger, J., Fischer, A., Bushati, N., Poernbacher, I., Mechtler, K., Cohen, S.M., and Knoblich, J.A. 2008. Mei-P26 regulates microRNAs and cell growth in the Drosophila ovarian stem cell lineage. Nature 454: 241-245.

Niki, Y. and Mahowald, A.P. 2003. Ovarian cystocytes can repopulate the embryonic germ line and produce functional gametes. Proc. Natl. Acad. Sci. 100: 14042-14047.

Nystul, T. and Spradling, A.C. 2007. An epithelial niche in the Drosophila ovary undergoes long range stem cell replacement. Cell Stem Cell 1: 277-285.

Ohlstein, B. and Spradling, A.C. 2006. The adult Drosophila posterior midgut is maintained by pluripotent stem cells. Nature 439: 470-474.

Ohlstein, B. and Spradling, A.C. 2007. Multipotent Drosophila intestinal stem cells specify daughter cell fates by differential Notch signaling. Science 315: 988-992.

Pan, L., Chen, S., Weng, C., Call, G., Zhu, D., Tang, H., Zhang, N., and Xie, T. 2007. Stem cell aging is controlled both intrinsically and extrinsically in the Drosophila ovary. Cell Stem Cell 1: 458-469.

Park, J.K., Liu, X., Strauss, T.J., McKearin, D.M., and Liu, Q. 2007. The miRNA pathway intrinsically controls self-renewal of Drosophila germline stem cells. Curr. Biol. 17: 533-538.

Snapp, E.L., Iida, T., Frescas, D., Lippincott-Schwartz, J., and Lilly, M.A. 2004. The fusome mediates intercellular endoplasmic reticulum connectivity in Drosophila ovarian cysts. Mol. Biol. Cell 15: 4512-4521.
Song, X., Wong, M.D., Kawase, E., Xi, R., Ding, B.C. McCarthy, J.J., and Xie, T. 2004. Bmp signals from niche cells directly repress transcription of a differentiation-promoting gene, bag of marbles, in germline stem cells in the Drosophila ovary. Development 131: 1353-1364.

Spradling, A.C., de Cuevas, M., Drummond-Barbosa, D., Keyes, L., Lilly, M., Pepling, M., and Xie, T. 1997. The Drosophila germarium: Stem cells, germ line cysts and oocytes. Cold Spring Harbor Symp. Quant. Biol. 62: 25-34.

Szakmary, A. Cox, D.N., Wang, Z., and Lin, H. 2005. Regulatory relationships among piwi, pumilio, and bag-ofmarbles in germline stem cells in the Drosophila ovary. Curr. Biol. 15: 171-178.

Tootle, T.L. and Spradling, A.C. 2008. Drosophila Pxt: A cyclooxygenase-like facilitator of follicle maturation. Development 135: 839-847.

Wallenfang, M.R., Nayak, R., and DiNardo, S. 2006. Dynamics of the male germline stem cell population during aging of Drosophila melanogaster. Aging Cell 5: 297-304.

Wang, L., Li, Z., and Cai, Y. 2008. The JAK/STAT pathway positively regulates DPP signaling in the Drosophila germline stem cell niche. J. Cell Biol. 180: 721-728.

Xie, T. and Spradling, A.C. 2000. A stem cell niche maintaining germ cell production in the Drosophila ovary. Science 290: 328-330.

Yamashita, Y.M., Mahowald, A.P., Perlin, J.R., and Fuller, M.T. 2007. Asymmetric inheritance of mother versus daughter centrosome in stem cell division. Science 315: 518-521. 


\section{$\$_{\text {CSH\& }}^{\infty}$ Cold Spring Harbor Symposia SYMPOSIA}

\section{Stem Cells and Their Niches: Integrated Units That Maintain Drosophila Tissues}

A.C. Spradling, T. Nystul, D. Lighthouse, et al.

Cold Spring Harb Symp Quant Biol 2008 73: 49-57 originally published online November 6, 2008 Access the most recent version at doi:10.1101/sqb.2008.73.023

References This article cites 45 articles, 20 of which can be accessed free at: http://symposium.cshlp.org/content/73/49.full.html\#ref-list-1

\section{License}

Email Alerting Receive free email alerts when new articles cite this article - sign up in the Service box at the top right corner of the article or click here. 